\title{
The role of the styloid apophysis of the temporal bone in the biomechanics of the tongue, mandible, hyoid system: a case study
}

\author{
Giuseppe Messina
}

(1) Department of Psychological, Pedagogical and Educational Sciences, Sport and Exercise Sciences Research Unit, University of Palermo, Palermo, Italy

This article is distributed under the terms of the Creative Commons Attribution Noncommercial License (CC BY-NC 4.0) which permits any noncommercial use, distribution, and reproduction in any medium, provided the original author(s) and source are credited.

\begin{abstract}
Temporomandibular disorders are characterized by ear pain, tinnitus, difficulty in chewing, dysphagia and also dizziness, cervical limitation of range of movement (ROM) and other less common disorders. Numerous recent studies associate the presence of these signs and symptoms with an elongated styloid apophysis, as in the case of Eagle syndrome. The purpose of this clinical case is to support the thesis of a common syndrome that temporomandibular joint (TMJ) disorders with an elongated styloid process affirming the relationship between temporal bone, mandibule, hyoid bone and tongue. The patient was a 57-year-old woman who reported having ear algia and tinnitus all on the right side with difficulty chewing accompanied by odynophagia and dysphagia of solid and liquid foods, as well as cervical disorders. Computed tomography (CT) scans of the neck showed elongation and ossification of the styloid processes of the temporal bone. Magnetic resonance imaging (MRI) showed an anterior right dislocation of the TMJ articular disc. The patient presented classic symptoms of temporomandibular disorders, with the primary examinations showing an anterior right dislocation of the articular disc of the TMJ, as well as a longer styloid process on the same side. We therefore suggested that, in this case report, TMJ disorders may be associated with a longer styloid process on the same side.
\end{abstract}

Key Words: TMJ disorders, Eagle syndrome, styloid process.

Eur J Transl Myol 30 (1): xx1-xx5, 2020

\begin{abstract}
As previously described, in a recent case report, the tongue, mandible and hyoid bone together constitute a single system. ${ }^{1}$ This may be seen by considering the functional interactions between these elements. For example, it has been shown that exercises with the lingual musculature, ${ }^{2}$ as well as the use of devices for the correct repositioning of the tongue, ${ }^{3}$ have a positive effect on the hypertonicity of the levator muscles of the jaw. Therefore, they can have benefits on the symptoms of temporomandibular joint (TMJ) dysfunction. The tongue-mandible-hyoid (TMH) system also plays a key role in the vital function of swallowing, therefore also assisting all mandibular movements, ${ }^{1}$ such as chewing and phonation. Embryologically, they derive from the cartilages of the II pharyngeal arch (innervation from the VII cranial nerve), both the styloid apophysis of the temporal and the small horn of the hyoid bone, together with the styloid ligament. There are three main muscles which insert into the styloid apophysis of the temporal bone. Their names, origin as well as innervation and action are shown below in Table 1. The three muscles, together with the two ligaments (stylomandibular and
\end{abstract}

stylohyoid), constitute the "Riolano bouchet". ${ }^{4}$ They are linked both functionally and anatomically. In the functional manner, they contribute with the other muscles and ligamentous structures of the TMH System to the mandibular movements. These include: phonation, and mastication and in the vital function of swallowing. Anatomically, the stylohyoid muscle divides into two parts, creating an ostium through which the intermediate tendon of the digastric muscle passes (muscle that inserts precisely into the digastric dimple of the jaw). This creates a functional syncytium: an interdependence that further links the styloid apophysis, hyoid bone and mandible. ${ }^{5}$ It can be thus hypothesized that these three muscles and the two ligaments (constituting the "Riolano bouchet"), which connect the styloid apophysis of the temporal bone to the tongue, to the mandible, to the hyoid bone and to the pharynx, are fundamental for the biomechanical stabilization of swallowing. This action occurs through a fulcrum arrangement, where there is a fixed point on the skull bilaterally, as the mandible and hyoid bone are suspended skeletal segments put in a parallel way. Therefore, the styloid apophysis of the 
Biomechanics of the tongue, mandible, hyoid system

Eur J Transl Myol 30 (1): xx1-xx5, 2020

Table 1. Origin, action and innervation of the three main muscles which insert into the stylohyoid apophysis.

\begin{tabular}{|l|l|l|}
\hline Muscle & Embryological Origin & Innervation and Action \\
\hline Stylohyoid & $\begin{array}{l}\text { Migration of cells from the sixth } \\
\text { somitomere to the pharyngeal arc II. }\end{array}$ & $\begin{array}{l}\text { Innervation by the VII pair of cranial } \\
\text { nerves, with action of elevation of the } \\
\text { bone hyoid in swallowing. }\end{array}$ \\
\hline Stylopharyngeal & $\begin{array}{l}\text { Migration of cells from the seventh } \\
\text { cranial somitomer to the third } \\
\text { pharyngeal arch. }\end{array}$ & $\begin{array}{l}\text { Innervation from the IX pair of cranial } \\
\text { nerves, with elevation of the pharynx. }\end{array}$ \\
\hline Styloglossus & Derived from the occipital somites. & $\begin{array}{l}\text { Innervation from the XII pair of cranial } \\
\text { nerves, with the action on the tongue of } \\
\text { elevation and posterior movement. }\end{array}$ \\
\hline
\end{tabular}

temporal bone, with the "Riolano bouchet", can be considered an integral part of the TMH system, which may also be described using an extended definition of the temporal-mandible-tongue-hyoid (TMTH) System

The clinical observation of patients with mixed symptoms,${ }^{6}$ and with Costen, ${ }^{7}$ and Eagle syndrome, ${ }^{8}$ led to a diagnostic imaging protocol, which included both magnetic resonance imaging (MRI) of the TMJ and the computerized tomography (CT) images of the styloid apophysis. ${ }^{9}$ This protocol demonstrated, in all the cases considered, a close correlation between the affected side and an increased length of the apophysis (with or without calcification of the styloid ligament) and with anterior dislocation of the articular disc of the TMJ. ${ }^{10}$ The aims of this study were to describe how a case involving a variety of symptoms, characteristics of TMJ disorders, prevalent in one side, are linked to an increased length of the apophysis and with anterior dislocation of the articular disc of the TMJ of the same side concerned. This work has led to the proposal of a "TMTH System Syndrome", including signs and symptoms common to Costen Syndrome $^{7}$ and to Eagle Syndrome. ${ }^{8,11}$

\section{Case Report}

The patient was a 57-year-old woman who reported to have had anterior and posterior ear pain and tinnitus all in the right side. The patient also reported having difficulty chewing hard foods with dysphagia of solid and liquid foods. The subject had subjective vertigo and cervical ROM limitation. The symptoms had progressively worsened in last 5 years, in terms of intensity and frequency. The clinical examination showed: pain on palpation of the lateral region of the neck bilaterally, of the masseter muscle and of the greater pterygoid muscle on the right side. It also showed left mandibular deviation in opening with sign of the "right click", with hypomobility of the hyoid bone in swallowing. In addition, there was a positive test for a morphologically anomalous styloid apophysis. The patient had various therapies, patient had used non-
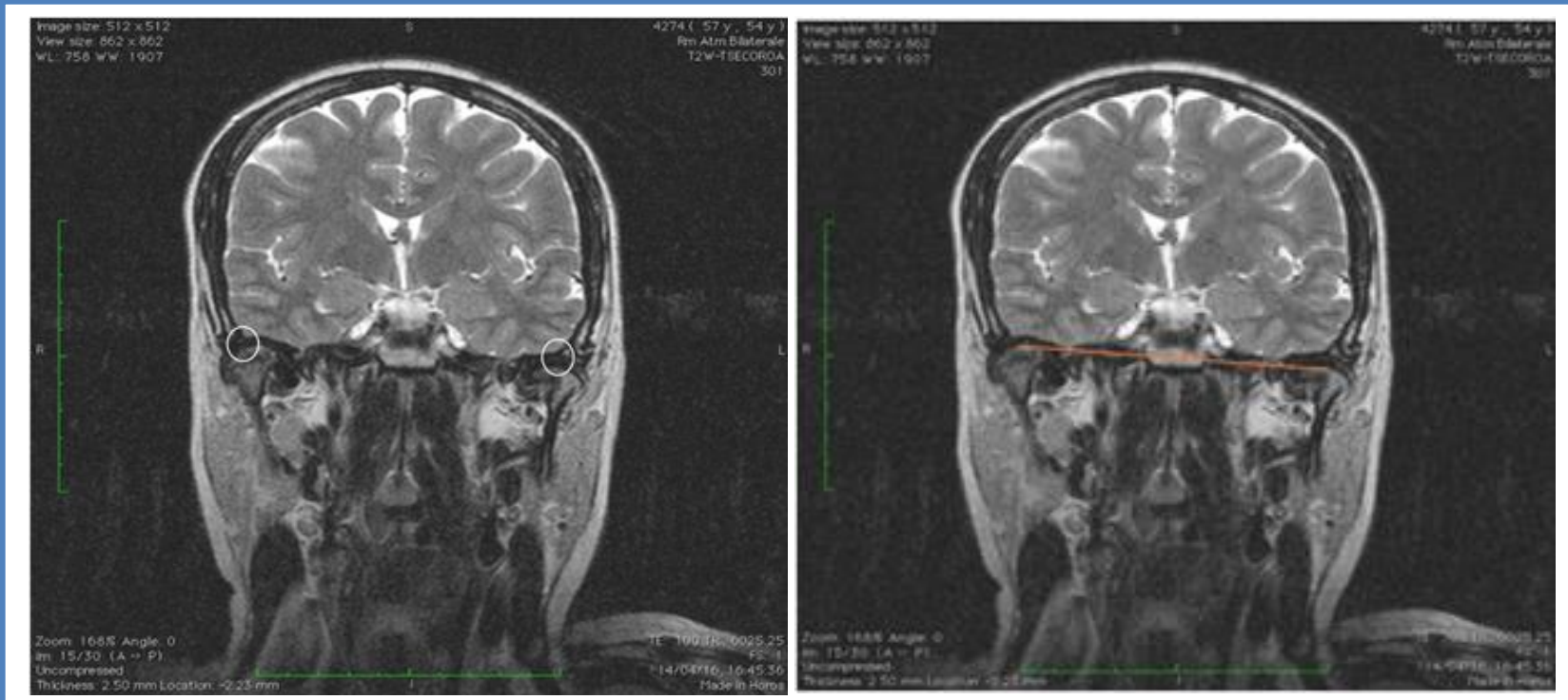

Fig 1. MRI, TMJ coronal scan T2W mouth closed: asymmetry (white circle in the left figure) of position of the condyles in relation to the right to anterior internal dislocation of the articular disc, with reduction of the upper-posterior TMJ joint space. 


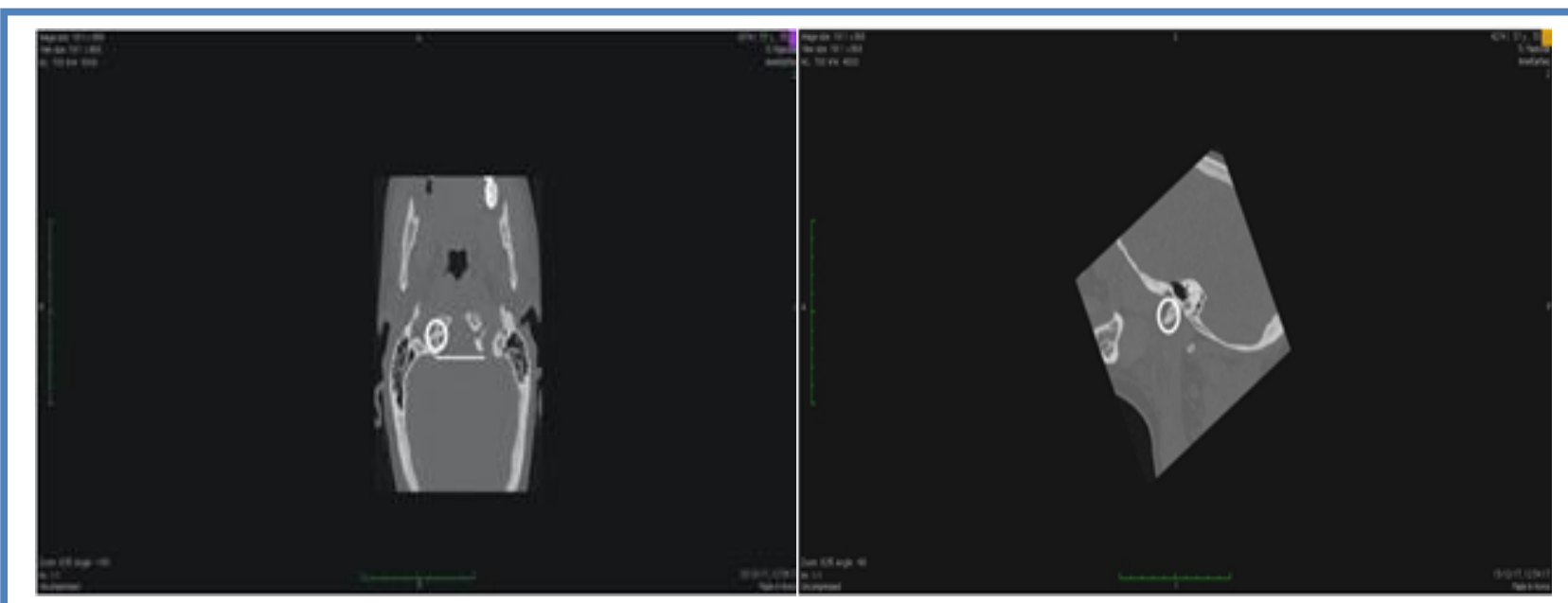

Fig 2. Left panel: Massive facial CT: alteration of the right styloid apophysis approximately $4.4 \mathrm{~cm}$ with asymmetry between the right and left styloid apophysis (white line in the figure) and calcification (white circle in the figure) of the styloid ligament insertion; Right panel: Massive facial CT scan, MPR2D reconstruction in the sagittal plane with elongation of right styloid apophysis (white line in the figure)

steroidal anti-inflammatory drugs (NSAIDs) before examination and had no other pathologies. Magnetic resonance imaging (MRI) showed an anterior right dislocation of the TMJ articular disc (Figure 1). The computed axial tomography (CAT) instead shows an elongated right styloid apophysis of $4.4 \mathrm{~cm}$, (Figure 2). ${ }^{11}$

\section{Discussion}

Recent studies have shown, ${ }^{12}$ that the symptoms of styloid process elongation may overlap with clinical manifestations of temporomandibular joint disorders explainable through muscle, tendon and nerve connections with the tongue, mandible and hyoid bone. Temporomandibular disorders (TMDs) are musculoskeletal pain conditions characterized by pain in the temporomandibular joint (TMJ) and/or mastication muscles. Patients with TMDs are frequently affected by otorhinolaryngological symptoms, but also TMJ disorders can cause impaired mobility of the cervical tract $^{13}$. In our case report the patient described these symptoms, most of which are almost exclusively unilateral, including the presence of tinnitus. In particular, for the latter, there is still a debate in the scientific community on the relationship between tinnitus and TMD. A possible explanation could be found in ontogenetic development and in the mechanical and neuronal connections of the masticatory system and the middle ear. Furthermore, a potential role of TMDs in the onset of tinnitus is suggested by the fact that the treatment of TMDs can successfully alleviate or treat these symptoms. ${ }^{14}$ The presence of tinnitus can also influence other functions such as postural control, auditory perception, and impeded balance. ${ }^{15}$ Subjects had altered stabilometry and baropodometry parameters, compared to the reference values of the standard population, which have been described in a later paper by Patti and colleagues in 2018. ${ }^{16}$ It is therefore essential to understand the origin and treatment of tinnitus to improve the quality of life for patients. In our case report, a correlation was found between the right symptomatic side (with TMJ symptoms described by the patient and evaluated at the clinical examination), with an abnormal length right styloid apophysis of $4.4 \mathrm{~cm}$ compared with the normal reference values. ${ }^{11}$ The normal styloid process measures are between 2.5 and $3 \mathrm{~cm} .{ }^{11}$ Furthermore, dislocation of the TMJ joint disc of the same side was also shown. Our data confirm what has been described in the literature: associating the main dysfunctional symptoms of the temporomandibular joint to those of Eagle syndrome, which depends on the abnormal length of the styloid, such as aspecific neck pain, pharynx, odynophagia, otalgia, pain in the trismus. ${ }^{17}$ This dysfunction would also seem to have greater prevalence in women, ${ }^{18,19}$ as in our case, and furthermore there seems to be a little correlation between the length styloid process and the severity of symptoms ${ }^{20}$. To date, computed tomography is considered to be the best examination for defining the length of the styloid process. ${ }^{21}$.

In this case study, we outlined the complexity of the muscles that connect to the tongue, jaw and hyoid bone and then described the temporal-mandible-tongue-hyoid (THTH) system. The muscles and ligaments that interconnect the tongue, jaw, hyoid and styloid hypophysis of the temporal bone form a biomechanical system that acts synergistically to produce important functions such as swallowing. The study presented in details a patient who had tinnitus, difficulty in chewing, dysphagia, vertigo and cervical ROM limitation. There was also pain in the neck muscle region, mandibular deviation and hyoid hypomobility during swallowing. The patient also had an abnormal morphology of the 
styloid apophysis. We argue that this case was similar to that of Eagle's Syndrome; and that a potential underlying driver of this condition is an abnormally elongated styloid process. The TMTH syndrome is defined on the basis of common elements between Eagle's and Costen's Syndrome. On the basis of this case report, we argue that in the clinical diagnostic phase of patients with TMJ disorders, it is advisable to check the possible presence of morphological alteration of the styloid apophysis, and/or the possible calcification of the stylohyoid ligament. In the therapeutic phase, ${ }^{2}$ the muscle tone of the TMJ muscles must be considered together with the normal function of the lingual muscles in swallowing. ${ }^{22}$ Furthermore, an evaluation of the physiological mobility of the hyoid bone should also be performed, considering a prerequisit the presence of asymmetry of muscle tone. The signs and symptoms manifested by anterior dislocation of the articular disc of the TMJ are related to an abnormal length of the styloid process of the same side affected by the disorders. We define this a temporalmandible-tongue-hyoid (THTH) Syndrome.

\section{List of acronyms}

CAT - computed axial tomography

CT - Computed tomography

MRI - Magnetic resonance imaging

NSAIDs - Nonsteroidal anti-inflammatory drugs

ROM - range of movement

TMDs - Temporomandibular disorders

TMH - tongue-mandible-hyoid

TMJ - temporomandibular joint

TMTH - temporal-mandible-tongue-hyoid

\section{Author contribution}

This is a single author report.

\section{Funding}

None

\section{Conflict of Interest}

Author declares no conflict of interests.

\section{Ethical Publication Statement}

We confirm that we have read the Journal's position on issues involved in ethical publication and affirm that this report is consistent with those guidelines.

\section{Corresponding Author}

Giuseppe Messina, Department of Psychological, Pedagogical and Educational Sciences, Sport and Exercise Sciences Research Unit, University of Palermo, Palermo I-90128, Italy

ORCID iD0000-0003-2774-4950

Email: giuseppe.messina17@unipa.it

\section{References}

1. Messina G. The tongue, mandible, hyoid system. Eur J Transl Myol 2017; 27 (1): 74-76.
2. Messina G, Martines F, Thomas E, et al. Treatment of chronic pain associated with bruxism through Myofunctional therapy. Eur J Transl Myol 2017 Jun 29;27(3):6759.

3. Rampello A, Papi P, Pompa et al. A novel universal device "LINGUAL RING Ri.P.A.Ra" for TMDs and cranio-cervico-mandibular pains: preliminary results of a randomized control clinical trial. European Review for Medical and Pharmacological SciencesIssue 5, 1180-1190.

4. Bumann A, Lotzmann U, Mah J. TMJ Disorders and Orofacial Pain: The Role of Dentistry in a Multidisciplinary Diagnostic Approach. New York, NY: Thieme Medical Publishers 2002:30.

5. Rathee M, Jain P. Anatomy, Head and Neck, Stylohyoid Muscle. StatPearls 2019.

6. Di Paolo C, Costanzo GD, Panti F, et al. Epidemiological analysis on 2375 patients with TMJ disorders: basic statistical aspects. Ann Stomatol 2013;4(1):161-9.

7. Parry LG. Costen's syndrome; a report of two cases. J Am Dent Assoc 1947;35:831-3.

8. Badhey A, Jategaonkar A, Kovacs AJA, et al. Eagle syndrome: a comprehensive review. Clin Neurol Neurosurg 2017.159:34-38.

9. Ayyildiz VA, Senel FA, Dursun A, Ozturk K. Morphometric examination of the styloid process by 3D-CT in patients with Eagle syndrome. European Archives of Oto-Rhino-Laryngology 2019. 276 (12): 3453-3459.

10. Costantinides F, Della Flora F, Tonni I, et al. Elongation of the styloid processes in kidneytransplanted patients: The role of ectopic calcification as possible cause of Eagle syndrome. Cranio 2019;11:1-5.

11. Eagle WW. Elongated styloid process: Report of two cases. Arch Otolaryngol 1937;25:584-546.

12. Zokaris N, Siska I, Natsis K, et al. Investigation of the styloid process length in a Greek population. Folia Morphol 2019;78(2):378-388.

13. Battaglia G, Giustino V, Iovane A, et al. Influence of occlusal vertical dimension on cervical spine mobility in sports subjects. Acta Medica Mediterranea 2016. Volume 32.

14. Sireci F, Salvago P, Messina G, et al. The role of temporomandibular disorders in the genesis of tinnitus. In: The Watson J, eds Tinnitus: Epidemiology, Causes and Emerging Therapeutic Treatments 2016. pp 1-10.

15. Martines F, Messina G, Patti A, et al. Effects of tinnitus on postural control and stabilization: a pilot study. Acta Medica Mediterranea 2015; 31: 907.

16. Patti A, Bianco A, Şahin N, et al. Postural control and balance in a cohort of healthy people living in Europe: An observational study. Medicine 2018; 97(52):e13835

17. Prasad KC, Kamath MP, Reddy JM, et al. Elongated styloid process (Eagle's syndrome): a 
Biomechanics of the tongue, mandible, hyoid system

Eur J Transl Myol 30 (1): xx1-xx5, 2020

clinical study. J Oral Maxillofac Surg 2002;60(2):171-5.

18. Beder E, Ozgursoy OB, Ozgursoy SK, Anadolu Y. Three dimensional computed tomography and surgical treatment for Eagle's syndrome. Ear Nose Throat J 2006;85:443-5.

19. Mendelsohn AH, Berke GS, Chhetri DK. Heterogeneity in the clinical presentation of Eagle's syndrome. Otolaryngol Head Neck Surg 2006;134:389-93.

20. Diamond LH, Cottrell DA, Hunter MJ, Papageorge M. Eagle's syndrome: a report of 4 patients treated using a modified extraoral approach. J Oral Maxillofac Surg 2001;59:1420-6.

21. Pinheiro TG, Rocha Soares VY, Lage Ferreira DB, Tet al. Eagle's Syndrome. Int. Arch. Otorhinolaryngol. 2013;17:347-50.

22. Messina G, Giustino V, Martines F, et al. Orofacial muscles activity in children with swallowing dysfunction and removable functional appliances. Eur J Transl Myol 2019;29:246-50.

Submission: January, 9, 2020

Revision received: February 12, 2020

Acceptance: February 12, 2020 